\title{
To our Referees
}

We are grateful to those who helped us review papers in 1973:

Alpert, E., Boston, Mass., USA Ambroise-Thomas, P., Grenoble, France Ambler, J.,

Loughborough, England Amdrup, E., Aarhus, Denmark Andersson, S., Stockholm, Sweden Andre, C, Lyon, France Andreasen, M., Copenhagen, Denmark Audigier, J.C., Lyon, France Baron, J. H., London, England Bengmark, S., Lund, Sweden Benhamou, J.P., Paris, France Bendixen, G., Copenhagen, Denmark Benscome, S.A., Kingston, Ontario,

Canada Benzi, G.M., Pavia, Italy Berard, P., Lyon, France Beutler, E., Duarte, Calif., USA Bircher, J., Berne, Switzerland Blum, A., Zurich, Switzerland Bode, Ch., Marburg/Lahn, Germany Bolin, T.D., Sydney, Australia Bonfils, S., Paris, France Bouchier, I.A.D., Dundee, Scotland Boyer, J., Chicago, Ill., USA Bralow, S.P., Philadelphia, Pa., USA Brodie, D.A., North Chicago, Ill., USA Brodsky, W.A., New York, N.Y., USA Brooks, F., Philadelphia, Pa., USA Burtin, B., Paris, France Case, R.M., Newcastle upon Tyne,

England Chanarin, I., Harrow, England Chayvialle, J.A., Lyon, France Chey, W.Y., Rochester, N.Y., USA Coates, M.E., Berkshire, England Code, C.F., Rochester, Minn., USA Cohen, S., Philadelphia, Pa., USA Condon, J.R., London, England

Conn, H.O., West Haven, Conn., USA Conrad, M.E., Washington, D.C., USA Cotton, P.B., London, England Cowley, D.J., Manchester, England Crane, R.K., New Brunswick, N.J., USA Creamer, B., London, England Dawson, A.M., London, England Debas, H.T., Los Angeles, Calif., USA Delcourt, A., Bruxelles, Belgium De Graef, G., Bruxelles, Belgium Demole, M., Geneva, Switzerland Dhumeaux, D., Paris, France Doberneck, R.C, Albuquerque, N. Mex., USA Dolezel, J., Hamilton, Ontario, Canada Doni $\lambda$ ch, D., London, England Dowling, R.H., London, England Duthie, H.L., Sheffield, England Edwards, D.A.W., London, England Emås, S., Stockholm, Sweden Erlinger, S., Paris, France Fahrlander, H., Basel, Switzerland Fiasse, R., Leuven, Belgium Fields, J.B., Pittsburgh, Pa., USA Fitzgerald, D., Dublin, Ireland Forell, M.M., München, Germany Franckson, J.M.R., Bruxelles, Belgium Fujita, M., Tokyo, Japan Gallagher, N.D., Sydney, Australia Gelb, A.M., New York, N.Y., USA Gelpand, M.D., Seattle, Wash., USA Gillespie, I.E., Manchester, England Glass, G.B.J., New York, N.Y., USA Goebell, H., Ulm, Germany Goldberg, D.M., Sheffield, England Golding, P.L., Portsmouth, England Gregory, R., Liverpool, England

316

To our Referees

Grossmann, M.I., Los Angeles, Calif., USA Hadorn, B., Berne, Switzerland Haex, K., Leiden, Netherlands Hallen, J., Malmö, Sweden Hansky, J., Melbourne, Australia Harvey, R.F., Bristol, England Hubel, K.A., Iowa City, Iowa, USA Hunt, A.C., Plymouth, England Hunter, J., Cambridge, England Isenberg, J., Los Angeles, Calif., USA Ishii, Y., Tokyo, Japan Islami, A.H., Livingston, N.J., USA Isselbacher, K.J., Boston, Mass., USA Jarnum, S., Copenhagen, Denmark Javitt, N.B., New 
York, N.Y., USA Johnson, A.G., London, England Johnston, D., Leeds, England Jones, M.C., London, England Jones, G.M., Nottingham, England Kaess, H., Heidelberg, Germany Kaye, M.D., Denver, Colo., USA Kidder III, G.W., Baltimore, Md., USA Kim, Y.S., San Francisco, Calif., USA Klosser, P.J., Amsterdam, Netherlands Konturek, S.J., Krakow, Poland Kowalewski, K., Edmonton, Alberta, Canada Kowlessar, O.D., Philadelphia, Pa., USA Kvepsers, L., Rochester, Minn., USA Lambilliotte, J.P., Bruxelles, Belgium Langman, M.J.S., Nottingham, England Lieber, C.S., Bronx, N.Y., USA Lieberman, J., Duarte, Calif., USA Lin, M.T., Indianapolis, Ind., USA McGuigan, J.E., Gainesville, Fla., USA Mackay, I.R., Melbourne, Australia Maratka, Z., Praha, Czechoslovakia Martin, F., Dijon, France Masseyeff, R., Nice, France Matuchansky, C, Paris, France Mignon, M., Paris, France Minaire, Y., Lyon, France Nishioka, M., Ube, Japan Nordstrom, C, Lund, Sweden Olsson, R., Gothenburg, Sweden Painter, N.S., London, England

Palmer, E.D., Morristown, N.J., USA Pansu, D., Lyon, France Perrier, C.V., Geneva, Switzerland Pettingale, K.W., London, England Pfeiffer, C.J., Newfoundland, Canada Quittner, H., Little Rock, Ark., USA Reddy, B.S., New York, N.Y., USA Rehfeld, J.F., Copenhagen, Denmark Revillard, J.P., Lyon, France Reynolds, T.B., Los Angeles, Calif., USA Ribet, A., Toulouse, France Robert, A., Kalamazoo, Mich., USA Rodgers, J.B., Albany, N.Y., USA Rosensweig, N.S., New York, N.Y., USA Rubin, E., New York, N.Y., USA Ruoscahti, E., Helsinki, Finland Sachs, G., Birmingham, Ala., USA Saries, H., Marseille, France Schaffner, F., New York, N.Y., USA Scheuer, P.J., London, England Schjonsby, H., Bergen, Norway Schneyer, L.H., Birmingham, Ala., USA Schofield, G.C., Clayton, Australia Schofield, B., Calgary, Alberta, Canada Schwartz, A., Houston, Texas, USA Scratcherd, T., Leicester, England Semenza, G., Zurich, Switzerland Sircus, W., Edinburgh, Scotland Siurala, M., Helsinki, Finland Sizaret, P., Lyon, France Smith, G.W., Baltimore, Md., USA Soltoft, J., Helierup, Denmark Spjut, J.H., Houston, Tex., USA Straub, R.W., Zurich, Switzerland Strohmeyer, G., Marburg/Lahn,

Germany Taylor, K.B., Palo Alto, Calif., USA Thayer, W.R., Providence, R.I., USA Tomasi, T.B., Buffalo, N.Y., USA Torrance, H.B., Manchester, England Torsoli, A., Roma, Italy Trépo, C, Lyon, France Truelove, S.C., Oxford, England Turner, M.D., Rochester, N.Y., USA Tuschka, O., Fresno, Calif., USA Tuyns, A.J., Lyon, France Unger, R., Dallas, Tex., USA

To our Referees

317

Vaerman, J.P., Bruxelles, Belgium Vagne, M., Lyon, France Van de Kamer, J.H., Zeist, Netherlands Venables, C.W., Newcastle upon Tyne,

England Warren, W.D., Atlanta, Ga., USA Webster, P.D., Augusta, Ga., USA Wegmann, T., St. Gallen, Switzerland Weser, E., San Antonio, Tex., USA

White, T.T., Seattle, Wash., USA Whitehead, R., Oxford, England Williams, R., London, England Witte, M.H., Tucson, Ariz., USA Woodley, J.F., Galway, Ireland Wormsley, K.G., Dundee, Scotland Wright, R., Southampton, England Zamcheck, N., Boston, Mass., USA 